\title{
THE REVERSIBLE SOL-GEL TRANSFORMATION
}

S. C. BRADFORD, B.Sc.

The numerous theories of gel structure fall naturally under three heads. The first class of one-phase, or molecular systems, resembles the "super-cooled liquid" theory of glass, and has difficulty in explaining the loss of mobility which occurs on cooling. The second, or two-phase liquid-liquid, type suffers from the defect that no emulsions are known which have really the properties of gels. Moreover, mathematical analysis shows ${ }^{1}$ that this structure does not readily account for the elastic properties of gels. The third, or two-phase solid-liquid, type is the oldest and most natural. It suggests immediately that the almost infinite increase in viscosity which takes place, with slight change of temperature, during gelation is due to the gradual separation of solid from solution and appears to give ${ }^{2}$ a simple explanation of all the properties of gels.

In order to understand fully the reversible sol-gel transformation from this point of view, it seems necessary to know the true relation of sols to ordinary solutions. A gradual transition in properties from coarse suspensions through sols to true solutions is generally assumed. This does not readily suggest an explanation of the marked differences in behaviour to electrolytes which occur. It seems probable, however, that sols are related more closely to metastable solutions, the behaviour of supersaturated solutions to electrolytes does not appear to have been studied up to the present. Recent experiments on banded precipitates in gels appear to indicate that supersaturated solutions may be sensitive to electrolytes like sols.

Bechhold ${ }^{3}$ had previously mixed solutions of silver nitrate and ammonium chromate in the presence of varying amounts of ammonium nitrate and found that, with increasing ammonium nitrate the precipitate of silver chromate gradually decreased, disappeared, and then gradually increased again. A similar effect was found with potassium sulphate instead of ammonium nitrate. The experiments indicate both a change in the actual solubility of silver chromate and in the metastable limit, since concentrations could be found at which a precipitate was produced only after standing. With citric acid, as a trivalent anion, there was an enormous increase in the value of the ionic product at which precipitation of silver chromate occurred, which varied with the amount of citric acid. When the metastable limit was reached a large excess of chromate was deposited. Experiments in gels indicated a similar effect in the case of potassium citrate and soluble phosphates. The effect may be like that of the hydrogen ion, which tends to prevent the precipitation of positive sols, or of the aluminium ion, which changes the sign and increases the stability of gold sols. The experiments are being continued. So far they afford evidence of considerable sensitiveness to electrolytes on the part of ordinary supersaturated solutions.

If this be so, suspensoid sols would correspond more closely to metastable solutions and emulsoid sols to true solutions. Indeed the characteristic properties of colloid solutions may be due to one 
or both of two separate causes : supersaturation and particle size. From this point of view there should be substances which exhibit colloid properties merely on account of the large size of their molecules, or, of a chemical, or other, tendency to aggregation. Their sols would be comparatively little affected by electrolytes and might be treated as an extreme case of ordinary solutions.

Glutin and $\delta$-galactan appear to fall in this class. The molecular weight of gelatine has recently been estimated ${ }^{13}$ at about 10,000 , which would give a molecular diameter of $1.75 \mu \mu$. Its rate of diffusion is very low and, consequently, its velocity of crystallisation or separation from solution. From von Weimarn's formula such bodies should form sols on heating and gels on cooling.

For, if such a substance of very low diffusivity be treated with a liquid in which it is only slightly soluble, the Nernst-Noyes formula for the velocity of solution, $\mathrm{V}=\frac{\mathrm{D}}{\delta} \mathrm{S}(\mathrm{L}-c)$, shows that, on heating, solution would take place slowly and the sol stage persist. The velocity of crystallisation, on cooling, would also be very small, so that a large excess concentration would be generated, and, if $v$. Weimarn's formula, $N=K \frac{P}{L}$, applies, $P$ would become great, $L$ small and $\mathrm{K}$ a maximum, so that $\mathrm{N}$ would be very great and a gel would be formed.

As has been .shown, the formula is open to criticism as being merely empirical and the meaning of certain terms somewhat indefinite. And it has been suggested that the equation might preferably be written

$$
\mathbf{N}=\mathrm{U} \cdot \frac{\mathrm{P}}{\mathrm{L}}
$$

where $U$ is a function, to be determined, of the viscosity of the reaction medium and of the size and structure of the particles in solution, whether due to physical aggregation or to chemical complexity. The experimental evidence shows that, as $\mathrm{N}$ increases, the precipitate passes through stages in which it appears as (1) large complete crystals only after some years, (2) ordinary crystals in a short time, (3) growth figures or needles, (4) amorphous, frequently showing spherical grains, or spherites, under the microscope, and (5) as a gel which cannot be differentiated by present-day optical instruments. The formula, therefore, offers a reason for the occurrence in the colloid form of such substances as those considered above.

By inoculation of supersaturated solutions, von Weimarn has demonstrated that in every stage, except the two extremes, the solid phase is crystalline; from which he infers that it is also crystalline in sols and gels. Debye \& Scherrer's ${ }^{5}$ X-ray analysis confirms this in a number of cases. The special case of gelatine will be discussed below.

Almost all the phenomena of gelation indicate a connection with the process of crystallisation. The properties of supersaturated solutions have frequently been observed in the case of both suspensoids and emulsoids. Gold separates spontaneously from sols of more than a certain concentration, and the precipitation is hastened by the addition of a few drops of a second solution of colloid gold. Gelatine gelates more quickly if solid gelatine be added. Rubber emulsions behave in the same way. The variation of the gelation temperature with concentration ${ }^{6}$ strongly suggests crystallisation. And this is supported by the temperature increase in the concentration of solid remaining dissolved in the mother liquor expressed from gels. ${ }^{7}$ The crystall- 
isation theory removes the difficulty of explaining the viscosity changes in emulsoid sols in accordance with Einstein's formula, since the changes are due to variation in the number of particles as well as their size. The osmotic pressure changes must be due to the same cause.

Moreover, there are numerous organic substances known which behave exactly as indicated by von Weimarn's formula, and can be obtained as desired in the ordinary crystalline, or in the gel, form. The sodium salt of acrylic acid crystallises from dilute solution as lenticular needles, while the boiling saturated solution sets to a "structureless" jelly. This seems to be due to a chemical tendency to aggregation. Examples could be multiplied indefinitely.

Except, therefore, that they are not readily obtained in the crystalline form, there seems to be no reason for supposing that gelatine and agar are exceptions to the rule. Gelating 2 per cent. gelatine ${ }^{8}$ shows gradual development of submicrons from four or five, showing Brownian movement, in a square division of the field, with a side of $9 \mu$, to 80 to 100 at rest in the same area. If the liquid be expressed from the gel, the submicrons are also visible ultramicroscopically ${ }^{19}$. In the case of reversible gels of 5-dimethylaminoanilo-3 : 4-diphenylcyclopentene-1:2-dione, ${ }^{10}$ the ultimate particles were visible as microscopic spherites of $10 \mu$ diameter.

It is therefore to be expected that, by suitable modification of von Weimarn's factors, as, for instance, by reduction in the value of $P$, it should be possible to obtain the ultimate particles of gelatine of a microscopic size. Although the experiments are still in a preliminary stage the results strongly confirm the theory. In the first experiments, already described, 110.3 and 0.5 per cent. gelatine, sols were allowed to evaporate spontaneously at room temperature. Thoy were only slightly opalescent at first, but developed, within a few days, an extraordinarily dense opalescence with the eventual deposition of a slight buff-coloured precipitate which was seen to consist of spherites up to about $2 \cdot 8 \mu$ in diameter. Except for the presence of bacteria, the appearance was much the same whether or not $0 \cdot 1$ per cent. mercuric chloride was present as antiseptic. Since the opalescence first appeared within a few hours, decomposition of the gelatine is unlikely. And it was found that warm 4 per cent. gelatine sols do not give a precipitate on standing with saturated mercuric chloride solution. The precipitate must therefore be due to gelatine which has been obtained of microscopic size in accordance with theory.

Experiments under aseptic conditions gave comparable results. Warm 0.4 per cent. gelatine sols were filtered into clean test-tubes, the liquid boiled and the ends of the tubes immediately drawn out and sealed. These similarly, though more slowly, developed an opalescence. In three weeks it had become almost opaque and at the same time the opalescence was observed to be settling down in the tube (Fig. 1). Assuming a density of $1 \cdot 16$ for the falling particles, the rate of fall, $14.9 \mathrm{~mm}$. in 37 days, corresponded to a diameter of about $0 \cdot 1 \mu$. The microscopical appearance, six weeks later, was that of innumerable grains just too small to be distinguished separately. But a fair number of larger grains were present up to about $0 \cdot 6 \mu$. In another experiment $0 \cdot 4$ per cent. gelatine, in small test-tubes, was allowed to evaporate at room temperature over concentrated sulphuric acid contained in larger tubes, which were sterilised and sealed. The liquid became turbid and half gelatinous, and had a similar microscopic appearance to that in the previous experiment, though with fewer larger particles 


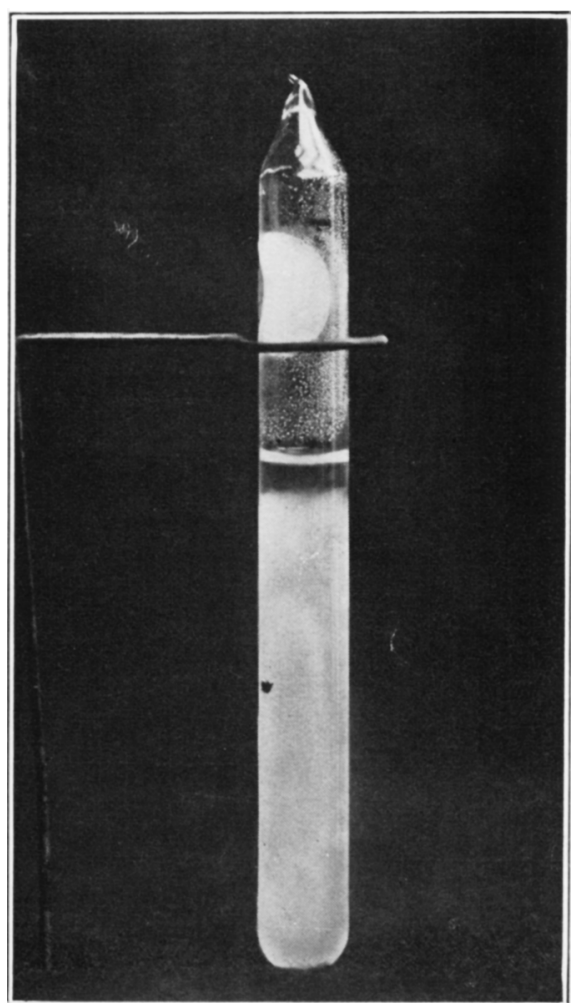

Fig. 1. 
$\mathrm{N}$ may also be decreased by increasing $\mathrm{L}$ by working at higher temperatures. Spontaneous evaporation of 5 per cent. gelatine sols at $31^{\circ}$ for 78 hours produced a smoky-looking jelly, which was still viscous in parts, but set on cooling to a stiff mass. The microscope showed this to be full of grains, which were just visible even with a 1-inch objective. Working at $40^{\circ}$ gave similar results. With a magnification of 200 the grains appeared perfectly spherical up to about $1 \mu$ in diameter. Many of these were aggregated into clusters somewhat like bunches of grapes. With crossed nicols a glowing point could be made out in the centres of some of the grains. In these experiments camphor was used as antiseptic.

There is, therefore, good reason for regarding the reversible sol-gel transformation as an extreme case of crystallisation; due to the very small diffusivity, and consequent minimum velocity of crystallisation, of the gel substance. With falling solubility on cooling, this necessitates the accumulation of an enormous excess concentration, with great increase of viscosity, and gives a maximum value of $\mathrm{N}$ in von Weimarn's formula corresponding to the gel state.

It has already been shown ${ }^{2}$ that this view is compatible with all the known properties of gels, as well as indicating new facts. The ultimate crystalline particles of different gels may differ in form according to the nature of the gel substance. The actual structure in a given case is difficult to determine. There are, however, certain phenomena which give some indication of the form of the gelatine particles. It has been shown ${ }^{2}$ that complicated organic substances tend to crystallise in the form of spherites. Regarded as an extreme case of twinning, this is obviously the form in which a body can most quickly be deposited. Von Weimarn's experiments show that, as the dispersity of the precipitate increases, the last stage in which the individual grains can be recognised is frequently that of spherical particles. In the experiments described above gelatin always appears in the spherical form. It seems likely that in the gel state the particles of gelatine are also of the same form. This agrees with the gradual increase in the number of discrete particles viewed ultramicroscopically in the gelating sol, and with the ultramicroscopic appearance of the resulting gel. It is further supported by the lenticular shape of gas bubbles in the gel. ${ }^{12}$.

Since spherites consist of a great many radiating filaments, if it be assumed that the deposition of solid gelatine occurs when the particles have reached a certain size, it would be expected that, when this limiting size should be reached, the filaments would hardly have attained a cross-section greater than one or two molecules. Such a structure would not give two adjacent planes, in the space lattice of the gelatine molecules, which could give interference of mono-chromatic $\mathrm{X}$-rays, which agrees with Scherrer's results. Or, it is possible that the lack of interference might be due to the great size of the gelatine molecule.

The above considerations suggest a structure for gelatine gels comparable to that of a pile of shot, but, as previously pointed out, ${ }^{2}$ they leave open the question as to whether the spherites are themselves united into a framework. The problem is complicated by the fact that the skins of gelatine gels show elastic properties greater than those exhibited by the internal jelly. The formation of the skin is probably due to further precipitation at the surface, due to adsorption and evaporation, so that the gel particles become felted and cemented together. It seems unlikely, therefore, that a gel which had been dried throughout, 
as in the case of ordinary sheet gelatine, would return to its original state by soaking in water. Consequently it is desirable to use freshly prepared gels in experimental work. Almost all the experimental results quoted in favour of a network within the gel could be explained by the presence of the skin, as, for instance, the gradual squeezing out of liquid from a swollen gel. ${ }^{13}$ Moreover certain facts appear to indicate that the particles in the interior of the gel are not always united, or are at least easily separated. The lenticular shape of gas bubbles has already been quoted. A gelatine cylinder, hung up by one end in a moist atmosphere, tends to become pear-shaped. Gelatine gels contract in the direction of greatest length when moderately heated, owing to the expansion of the gel within its skin. ${ }^{2}$ A gelatine cylinder diminishes in breaking stress on cutting the skin. Three cylinders of 5 per cent. gelatine 24 hours old showed breaking stresses $\frac{\text { (gram) }}{\left(\mathrm{cm}^{2}\right)}$ of respectively $16 \cdot 9,25$ and 25 for the interior gel, and 33,66 and 133 for the skin, giving a ratio varying from $2 \cdot 1$ to $5 \cdot 3$. On the other hand, aggregation must be assumed in the case of the skin, and insufficient concentration is the only reason why the particles should not grow together within the gel. Crystallisation experiments show a marked tendency of the spherites to aggregation. The remarkable behaviour of the 0.4 per cent. gelatine sols sealed in test-tubes somewhat supports this view. The tubes were not kept in a thermostat and showed a gradual increase in rate of falling of the particles, which may have been due to increase in size. After about 40 days the room temperature was lower and the rate of fall of the particles had diminished considerably. A little later it was observed that the cloudiness below the clear liquid, appeared to have set to a gel, which seemed to be just coherent, but broke up on inverting the tube.

The Science Museum, S.W.7.

\section{References}

1 Hatschek, Trans. Farad. Soc, 1917, $12,17$.

2 Bradford, Biochem. J., 1918, 12, 351.

3 Bechhold, Zeitsch. physikal. Chem., 1905, 52, 185.

4 Bradford, Biochem. J., 1920, 14, 474.

- Debye \& Scherrer, Physikal. Zeitsch., 1916, 17, 277; Scherrer, Nachr. Ges. Wiss, Göttingen, 1918, 96-100, etc.

- Pauli and Rona, Beiträge, 1902, 2, 4; Rohloff, Physikal. Zeitschr., 1907, 8, 442, and Levites, Kolloid. Zeitschr., 1908, 2, 162, 240.

7 Hardy, Proc. Roy. Soc., A. 1900, 65, 95.

senz, Zeitsch. physical. Chem., 1909, 66, 137.

- Bachmann, Zeitsch. anorg. Chem., 1911, 73, 150.

10 Hardy, Proc. Roy. Soc. A., 1912, 87, 29.

11 Bradford, Biochem. J., 1920, 14, 91.

12 Hatschel, Koll. Zeitschr., 1914, 15, 226; Bradford, Biochem. J., 1918, 12, 351 ; and Osborne, Proc. Roy. Soc. Victoria, 1918, 30, 153.

13 Lloyd, Biochem. J., 1920, 14, 147.

\section{Note added 29th October, 1920.}

The undisturbed gel resulting from the setting of the 0.4 per cent. gelatine solution, described above, was observed to contract beneath the clear liquid. The surface of the gel began to shrink away from the side of the tube, and, in seven days after setting, the whole of one side of the gel had come away from the tube, leaving a clear space of about $1 \mathrm{~mm}$. At the time of writing, the shrinkage was still going on. Since no attempt had been made to adjust the gelatine to the iso-electric point, the shrinkage is of interest in connection with a similar contraction currently desoribed (D. J. Lloyd, Biochem. J., 1920, 14, 584) of a 1 per cent. gel prepared from iso-electric gelatine. 